Old Dominion University

ODU Digital Commons

VMASC Publications

Virginia Modeling, Analysis \& Simulation Center

2015

Integrating Truck Emissions Cost in Traffic Assignment

Peter Foytik

R. Michael Robinson

Follow this and additional works at: https://digitalcommons.odu.edu/vmasc_pubs

Part of the Oil, Gas, and Energy Commons, Public Policy Commons, and the Transportation Commons 


\title{
Integrating Truck Emissions Cost in Traffic Assignment
}

\author{
Peter Foytik and R. Michael Robinson
}

\begin{abstract}
The adverse impacts of greenhouse gasses (GHG) and the imperative for reducing the existing rate of GHG production are well established. In the United States, the largest source of GHG emissions from human activities is from burning fossil fuels, primarily for the generation of electricity and transportation. The transportation sector accounts for $28 \%$ of all U.S. GHG production. Heavy-duty vehicles, such as large freight trucks, account for nearly one-fifth of the U.S. total, and this fraction is expected to grow rapidly. Consequently, many efforts are being used to reduce the total emissions of freight trucks. Most efforts emphasize one of four areas: engineering improvements to improve fuel economy or reduce emissions, shifts to other transport modes, improved logistics to reduce the movement of partially full or empty containers, and reduced travel costs for individual trucks. A few studies have assessed modifications to route choice considerations as a means of improving the fuel economy of individual vehicles and show potential gains. In this study, the potential gains of emissions-based route choice were assessed by integrating the U.S. Environmental Protection Agency motor vehicle emission simulator with a macroscopic regional traffic demand model. For this integration, route choices included a simplified emissions calculation within the repeated model iteration runs of an algorithm of the Frank-Wolfe type. The analyses suggested that reductions of freight truck emissions were possible and showed an example in which the total system's truck emissions were reduced by up to $0.61 \%$ (88.8 tons).
\end{abstract}

The adverse impacts of greenhouse gases (GHG) and the imperative to reduce the existing rate of GHG production are well established. In the United States, the largest source of GHG emissions from human activities is from burning fossil fuels, primarily for the generation of electricity and transportation. The transportation sector accounts for $28 \%$ of all U.S. GHG production, a value that exceeds the total GHG production from all sources by any other country in the world except China (1). Heavy-duty vehicles, such as large freight trucks, account for nearly one-fifth of the U.S. total and this fraction is expected to grow rapidly. Consequently, many efforts are in use to reduce the total emissions of freight trucks. Most efforts emphasize one of four areas: engineering improvements to improve fuel economy or reduce emissions, shifts to other transport modes, improved logistics to reduce the movement of partially full or empty containers, and reduced travel costs for individual trucks.

Virginia Modeling Analysis and Simulation Center, Old Dominion University, 1030 University Boulevard, Suffolk, VA 23435. Corresponding author: P. Foytik, pfoytik@odu.edu.

Transportation Research Record: Journal of the Transportation Research Board, No. 2503, Transportation Research Board, Washington, D.C., 2015, pp. 119-127. DOI: 10.3141/2503-13
A small fraction of studies have assessed modifications to route choice considerations as a means of improving the fuel economy, a value almost directly related to emissions, of individual vehicles. These studies suggest the potential gains, although valuable, are very small.

In transportation studies, equilibrium models are used to distribute traffic flows over road networks based on minimization of a measure of path cost usually involving the measurement of congested travel time. A common algorithm used for this traffic assignment step is usually the Frank-Wolfe algorithm. This algorithm will minimize path costs for the entire system iteratively until no new paths can be found to reduce the path costs of the total system. Traditionally, travel time is the measure of cost used, but tolls, the value of time, or other measures can be used to determine route preferences. Traffic assignment can also be used to reduce the total atmospheric emissions from the transportation system. Evaluations of the complete system benefit from the use of an iterative traffic assignment algorithm within the emissions calculation.

In this study, the potential gains of emissions-based route choice were assessed by testing a simplified measure of emissions within a regional macroscopic travel demand model. The emissions measure was updated and utilized as part of the path cost in a Frank-Wolfe algorithm. This measure of emissions is not intended to be a more accurate measure of emissions, but a relative measure that can be used to reflect paths of potentially reduced emissions based on the updated congestion results of the traffic assignment. The results were checked with the U.S. Environmental Protection Agency motor vehicle emission simulator (MOVES) as a way to verify potential emissions reduction. Analysis of an example showed reductions of freight truck emissions for an entire region of up to $0.61 \%$ (88.8 tons).

This research is an incremental contribution to the ongoing work of reducing transportation system emissions. It provides a method for consideration of total system emissions within the traffic assignment step. In doing so, it considers the total truck volume using the system, representing route choice at the regional scale rather than for individual trucks.

\section{LITERATURE REVIEW}

Reducing detrimental atmospheric emissions from the transportation system is a global imperative. Many action alternatives have been proposed, most of which emphasize one of four areas: engineering improvements to improve fuel economy or reduce emissions, shifts to other transport modes, improved logistics to reduce the movement of partially full or empty containers, and reduced travel costs for individual trucks. A sampling of these studies is provided here.

Dablanc et al. (2) reviewed 261 international references in an effort to identify the most effective strategies employed in other 
countries that could be adopted in the United States. The environmental impact programs focused on reducing truck emissions and energy consumption by improving engine performance, shifting to cleaner burning diesel trucks or alternative fuel trucks, or shifting freight to more energy efficient modes. Although improvements that use route choice alone were not evaluated, the results emphasized that strategies that address the entire commercial fleet have the most impact, even if the impact is small on a per vehicle basis. Similarly, Bühler and Jochem sought to reduce emissions associated with freight transport by shifting from heavy-duty trucks to other modes, such as rail and maritime vessels (3).

China is the most populous country in the world and one of the fastest-growing economies. Yan and Crookes (4) sought to analyze the future trends of energy demand and GHG emissions in China's road transport sector and to assess the effectiveness of possible reduction measures with detailed models of the historical energy demand trend and future demand forecasts. The proposed reduction efforts emphasized potential policy impacts, such as the imposition of fuel taxes and greater support for alternative fuels. Li et al. reported on efforts to reduce future transport-related emissions in China's largest city, Shanghai, by legislative action (5). These regulations can be categorized into five policy packages; none of the packages addresses the potential benefits that could be achieved by route choice methods.

Pietz and Gregor (6) authored a statewide study in Oregon to develop a long-term strategy for emissions reduction and control for all contributing sectors by the year 2050. Their report noted that freight proved the most challenging travel market to reduce overall emissions and the modeling projections show freight to be the largest emitting segment of the transportation sector in the future. Freight strategies, such as increasing operational efficiencies and technological improvements for cleaner fuels, were found to help reduce emissions, as did urban consolidation centers and true cost pricing, but substantial reductions were not predicted.

Bhagat et al. evaluated the potential reduction in emissions that could be achieved by shifting the times of freight movements from busier daytime periods to less congested nighttime periods (7). The PierPASS program was designed to reduce congestion and improve air quality. Although voluntary, the program imposed a fee on containers moved during regular working hours from Monday to Thursday. With data from PierPASS, a microscopic transportation simulation of container movements, was developed in TransModeler and integrated with MOVES to assess emissions. The researchers found that although much freight traffic was successfully shifted to off-hours, PierPASS had little impact on congestion in the study area and likely resulted in only a small overall decrease in the emissions of various air pollutants. Reductions in pollutants during daytime periods were offset by increased emissions of the same pollutants during off-peak hours.

Greene and Plotkin reported that technologies already in existence could reduce GHG emissions from freight trucks by $30 \%$ to $50 \%$, with even greater reductions achievable over the next several decades (1). Changes in vehicle operations could also provide improvement, although some might increase costs, making it unlikely that they will be seriously considered. Other operational changes, such as improving truck routing, avoiding empty return trips, consolidating shipments, and reducing truck idling, might actually improve profitability while reducing emissions. McKinnon conducted an analysis of the UK government's transport key performance indicators program, which benchmarks the efficiency of road freight operations (8). He found that the emphasis placed on fuel efficiency (essentially directly related to emissions rates for freight trucks) varied widely between different industries and even between companies within a given industry. Keys to this variance were requirements for just-in-time product deliveries, product perishability, and delivery environment (urban, highway, etc.). McKinnon also noted that companies could be reluctant to divulge some efficiency best practices for fear of losing commercial competitive edge (8). The latter finding supports the need for increased government participation in emissions reductions through operations guidance, including route choice.

Rowell et al. (9) participated in a Washington State Department of Transportation effort to characterize freight vehicle route choices to improve future decision making. A survey was conducted of approximately 800 shippers, receivers, and carriers of freight in Washington to identify strategies used in route choice. They found that off-theshelf routing software automatically assigns routes on the basis of least cost, given an origin-destination pair (determined by customer requirements), although selected paths can be filtered by other constraints. The survey also revealed that only a small minority of companies in Washington uses routing software and that route choice decisions were overwhelmingly driven by the desire to minimize costs (9).

Ahn and Rakha showed that ignoring acceleration impacts in emissions-based route choice algorithms could lead to the use of less beneficial arterial roads rather than highways (10).

\section{METHODOLOGY}

Traditional macroscopic models use emissions models in a postprocesses procedure, where the travel demand model calculates the vehicular flows over a road network based on a cost (usually travel time). After flows are produced, the loaded network is used as an input to the emissions model to process the amount of emissions that would be produced from the results of the travel demand model. To include a calculation of emissions within the traffic assignment step of the four-step process, the emissions calculation will need to be integrated within the iterative traffic assignment process. With calculation times of emissions models such as MOVES being very large, a full emissions calculation within the traffic assignment is not feasible. Instead, a relative emissions calculation is used based on a binning process and an aggregate vehicle-specific power (VSP) calculation. This relative emissions calculation is used within the iterative traffic assignment steps. The iterative emissions results are used as an updated value of cost between iterations of the traffic assignment.

MOVES was chosen as the emissions model used. MOVES is commonly used in macroscopic model emissions calculations. It provides calculations of important emissions for diesel long haul trucks and provides the means for reading in a loaded network and producing emissions rates based on the results. MOVES uses a binning strategy to determine emissions rates and provides access to its emissions database for use in custom calculations for the relative emissions calculation that will be utilized in the calculation within the traffic assignment step. In addition, MOVES is endorsed by the Environmental Protection Agency and its directed use is likely to continue.

The transportation model used for travel demand modeling is Citilabs Cube Voyager (11). Voyager was chosen because of its common use in the community as well as features that made it applicable to this research. The basic flow of the Voyager traffic assignment module Highway is shown in Figure 1. Highway provides a 


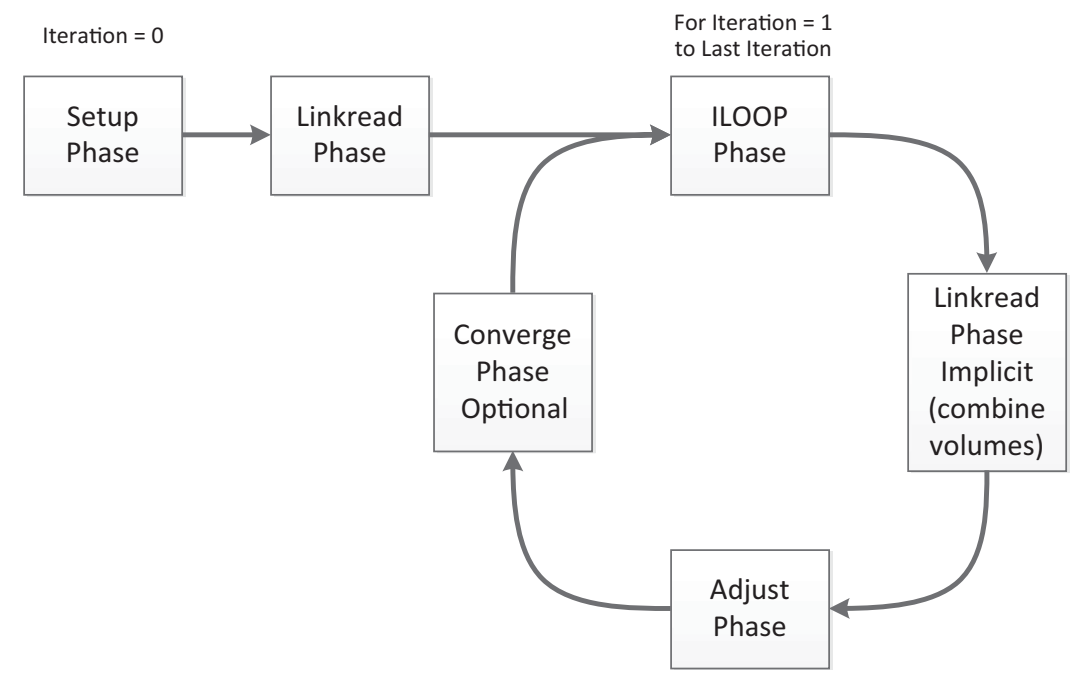

FIGURE 1 Flowchart for Cube Voyagers Highway module used for traffic assignment (11).

means to script functionality between traffic assignment steps with the use of the adjust phase. Highway also provides a converge phase where some processes could occur after new link travel times have been determined and before the next iteration. Cube also allows the execution of external applications and network postprocessing script to customize input files for MOVES, or to provide data for analysis. A Cube travel demand time-of-day model for the Hampton Roads area in southeastern Virginia was available and used in the study.

The Hampton Roads Travel Demand Model that was used is a complete four-step model of trip generation, trip distribution, modal split, and traffic assignment. The model does this process for two peak periods, a.m. and p.m., and two off-peak periods, midday and night. The model includes trips of single occupancy, two-person occupancy, and three-person occupancy, and trucks for varying levels of time. For this particular model, the midday demand modeled more truck trips than the other times of day. Because it had the larger truck demand, the midday model traffic assignment inputs were used as the basis for this research. The midday model modeled 37,225 trucks, just over $2 \%$ of the model's total volume.

The methodology to apply emissions within the traffic assignment algorithm requires that between traffic assignment iterations, the emissions for each segment are determined and stored for each road segment based on updated values of traffic flow and updated measures of congested travel time. The measure of emissions in the traffic assignment step will be based on the binned rates for carbon dioxide retrieved from the MOVES input database. The binned emissions rate for a segment can be determined by providing the segment's average speed and operation mode (OpMode). OpMode classification within MOVES is determined by the average speed and VSP.

Average speed can be derived from the congested travel time calculated within the traffic assignment, but VSP needs to be calculated separately. The calculation of VSP from the MOVES documentation is provided in Equation 1. In this calculation, vehicle speed, grade, and acceleration can be obtained from the model. The rest of the coefficients must be determined on the basis of the area modeled.

$\mathrm{VSP}=\left(\frac{A}{M}\right) * v+\left(\frac{B}{M}\right) * v^{2}+\left(\frac{C}{M}\right) * v^{3}+(a+g * \sin \theta) * v$ where

$$
\begin{aligned}
A & =\text { road load coefficient }(\mathrm{kW}-\mathrm{s} / \mathrm{m} \text {-tonne }), \\
B & =\text { road load coefficient }\left(\mathrm{kW}-\mathrm{s}^{2} / \mathrm{m}^{2} \text {-tonne }\right), \\
C & =\text { road load coefficient }\left(\mathrm{kW}-\mathrm{s}^{3} / \mathrm{m}^{3} \text {-tonne }\right), \\
M & =\text { mass of vehicle }(\mathrm{kg}) \\
g & =\text { acceleration due to gravity }\left(9.8 \mathrm{~m} / \mathrm{s}^{2}\right), \\
v & =\text { vehicle speed }(\mathrm{m} / \mathrm{s}) \\
a & =\text { vehicle acceleration }\left(\mathrm{m} / \mathrm{s}^{2}\right), \text { and } \\
\sin \theta & =(\text { fractional }) \text { road grade }
\end{aligned}
$$

Vehicle acceleration is not directly given and must be estimated to provide a more accurate measure of VSP. Flow-based macroscopic models provide flow for a static period of time, so acceleration will only be an estimate. Because the model does not provide dynamic flows, the estimate for acceleration comes from the change in average speeds from an upstream link to a downstream link. Therefore, a link's average speed is represented by

acceleration $=\frac{\left(\text { downstreamspeed }^{2}-\text { upstreamspeed }^{2}\right)}{\left(2 *\left(\frac{\text { distance }_{\text {normalizedvalue }}}{\text { nolized }}\right)\right)}$

Determining the distance becomes very difficult because macroscopic models represent an average speed for the length of a segment connected to the next segment, which instantly has an average speed measured for it. Because of this issue, a distance value is normalized based on the extreme case where a vehicle accelerated from the lowest speed to the highest speed, resulting in the highest OpMode. This process allows the measure of acceleration to provide reasonable results so that the methodology can be tested.

The speed of downstream segments is preprocessed with network scripts within Cube, where upstream links are provided values of downstream speed based on the average free-flow speed of all adjacent downstream segments. The updated values for downstream vehicle speeds cannot be retrieved in the same traffic assignment time step because of the timing when the updated travel time values are available. Because of this limitation, the current segment within 
the cost calculation utilizes an updated vehicle speed value and then uses the recorded downstream speed from the preprocessing. During the adjust phase shown in Figure 1, the script loops through every link and stores the updated congested travel time. With the downstream travel times and vehicle speed values for each segment, an acceleration value for that link can be calculated.

The acceleration value and updated speed of the road segment are used to obtain a rough measure of VSP and with that and the segment's average speed, an OpMode value can be determined. The OpMode value is then used to determine a mean base rate value from the input bin table. With the updated value for volume on the segment at the particular iteration of traffic assignment, the amount of emissions can be determined by multiplying volume by the mean emissions base rate. The resulting value can then be incorporated in the path cost for the next iteration of traffic assignment. The resulting measure of emissions is not intended to provide a better calculation of emissions, but a calculation that can be used in comparison to find better routes that minimize the total system emissions.

After traffic assignment has run, the loaded network provides the distribution of flows based on the cost value specified and can report the value of total emissions per iteration to demonstrate how well it is converging to a minimum. The final total emissions can be observed to get an idea of how much reduction in emissions occurred based on the calculation above. To test if emissions were reduced, the loaded output network is run through MOVES with its inventory calculation to determine a more accurate measure of total emissions from the model run.

The methodology was tested with truck volumes alone, allowing a better understanding of what the truck volume would do without the influence of car traffic. A traffic assignment is run for two scenarios: one in which trucks choose paths based on travel time (TT) and one with truck paths chosen based on TT plus emissions. The emissions results can be compared between the two scenarios and convergence can be observed to ensure that traffic assignment is working to minimize the system's total emissions. After the truck volume alone is tested, traffic assignment is set up to model truck flows and passenger car flows. Truck flows pick paths based on the congested segments' TT value plus the truck volume emissions calculation. Passenger cars use TT alone as the path cost. The combined truck and passenger car flow scenarios were run twice, first with truck flows utilizing a path cost of TT plus emissions and cars using TT, and then with trucks and passenger cars considering only TT. The emissions calculation for the combined car and truck scenarios will have the traffic assignment determine the minimization of emissions based on truck flows, but also inherently passenger car flows because the passenger cars will have an effect on the congested speed of the segments used by trucks. The truck flows could take paths that influence passenger cars to reroute. The final loaded networks are then used as inputs with MOVES to determine the amount of emissions produced by each scenario and if the methodology led to any reduction in emissions.

\section{RESULTS}

Traffic assignment was run for truck volume only with path costs set to congested TT plus emissions and for TT only. The purpose of the initial tests was to verify that the traffic assignment algorithm converged to a lower emissions value and behaved as intended, an incremental minimization of total system cost over the traffic assignment iterations. An extreme drop and then equilibrium was not preferred, as this could indicate that the cost is not flexible enough to allow the system to adapt out of a local minimum. The results that showed emissions increase and decrease without an average minimization behavior were not preferred, as this shows the system is unable to minimize toward equilibrium. Complete equilibrium was not needed for the first test, so only 10 iterations were run and results were observed.

After 10 iterations, the desired relative gap value was achieved. The relative gap shows how close the system is to equilibrium by showing the change in system cost between traffic assignment iterations (12). Because emissions contribute to the total system cost, the reduction in total system emissions should reflect the same shape as the change in relative gap per traffic assignment iteration. The results of the first run were unfavorable because emissions values were large in comparison with the TT value that the traffic assignment algorithm is meant to utilize. To achieve a more appropriate value of emissions cost in the traffic assignment algorithm, a flat factor was divided by the emissions value for the cost measurement. The true measure of emissions was recorded for comparative purposes.

With the reduction method for the emission cost value, the truck volume scenario was again run for 10 iterations. Figure 2 shows that as the relative gap is reduced (Figure $2 b$ ), the system's total emissions are also reduced (Figure $2 a$ ). Relative gap is a measure of the difference between two iterations; therefore, the first iteration does not contain a relative gap, and the first iteration of traffic assignment is an all-or-nothing assignment, meaning that unrealistic flows are distributed in the system based on free-flow speed, resulting in a very large relative gap value (and emissions value). Therefore, the first measure is disregarded so that the details of the chart can be seen.

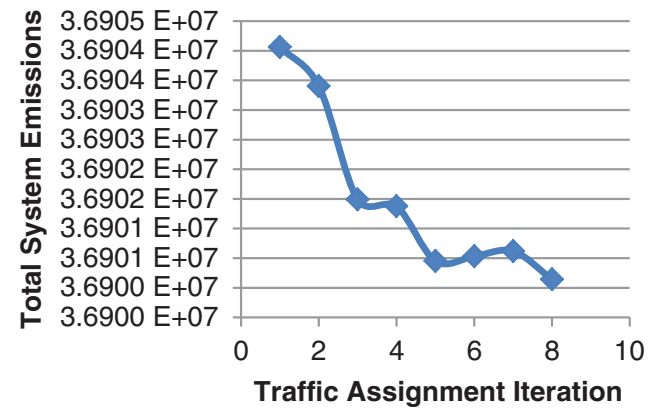

(a)

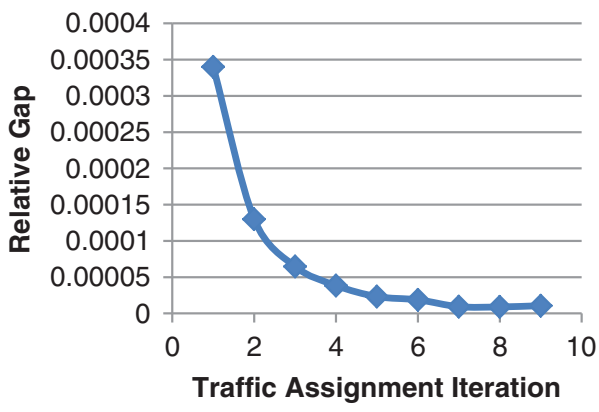

(b)

FIGURE 2 Truck volume scenario results per traffic assignment iteration with smaller measure of emissions for path cost: $(a)$ emissions reduction and $(b)$ relative gap reduction. 


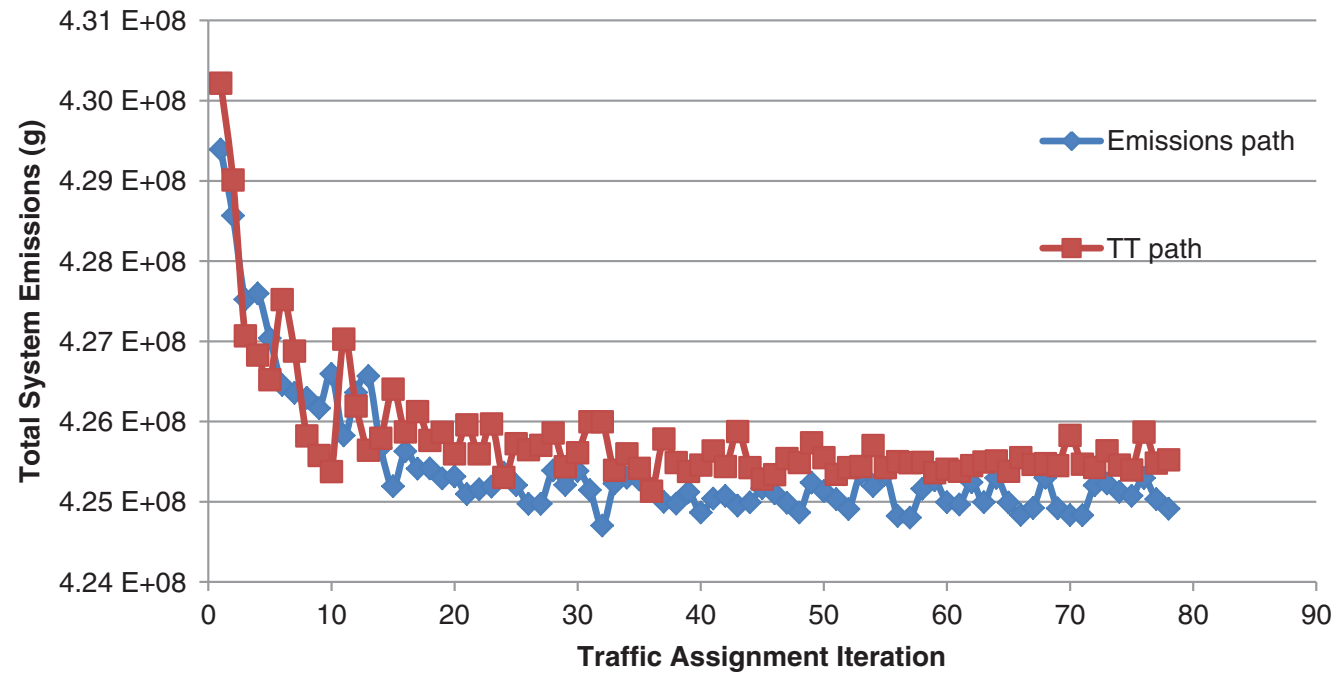

FIGURE 3 Comparison of total system emissions per traffic assignment iteration for scenario in which trucks use emissions in path cost and cars use travel time and scenario in which trucks and cars use travel time only for path costs.

The results for the truck-only scenarios confirmed that the methodology was working in that traffic assignment converged to a minimum system cost with emissions applied to the path cost. The next test was a comparison of the same scenarios with passenger car traffic along with truck traffic. A test run was done with 20 iterations and showed the system was still converging with a high relative gap remaining at the end. This result indicated that more traffic assignment iterations should be run to get the system closer to a state of equilibrium. Figure 3 shows the total emissions results per traffic assignment iteration from scenarios of trucks and passenger cars together. The emissions path series shows the total emissions per iteration, where trucks utilized a path cost including a weighted TT and emissions considerations and passenger cars used TT as their path cost. The TT path series shows the total emissions per iteration, where trucks and passenger cars utilized a path cost of TT only.

The results displayed in Figure 3 show that truck emissions reduce as the system's total TT is reduced. This finding suggests that total emissions could be reduced if emissions are considered in the path cost equation. The results also show that equilibrium was probably reached after approximately 60 iterations, as the change in values greatly reduced at that point. The actual amount of emissions that the system produced is predicted with the simplified calculation described in the methodology. But a better measure of the emissions will come from a true emissions model such as MOVES. The outputloaded networks are postprocessed so that they can be used as inputs to the MOVES model. MOVES calculates the total emissions for the system and the results for the MOVES runs and the traffic assignment runs are presented in Table 1.
These results report emissions values for the total system. The results from MOVES produced values of emissions much higher than the simplified binned version that was used in traffic assignment. The purpose of the simplified calculation used in traffic assignment was not to produce accurate emissions results, but to provide comparative results for emissions to allow the system to reduce total emissions. The three scenarios described are shown in the table, the traffic assignment of just truck volume, the traffic assignment of truck volume and passenger cars, and the MOVES results that use the results from the traffic assignment of trucks and passenger cars. The results show that the simplified emissions calculation provides a more relative and more conservative measure of emissions, as shown in the difference between the two scenarios of TT Paths and EMISS Paths. The traffic assignment showed $0.14 \%$ improvement of emissions and MOVES assessed a $0.61 \%$ (88.8 tons) improvement across the system. The improvements are small, but are represented for the entire system and represent a large amount of emissions. With a small change in emissions, the results indicate that routes that reduce congested travel time do a pretty good job at reducing emissions. If favorable emissions routes existed in the road network, truck volume would be more likely to occupy those routes instead routes that favor travel time.

The two scenarios produced different measures of emissions. Further investigation was completed to understand the rate at which trucks reroute to make these improvements. Consequences to TT are observed to accomplish this reduction in emissions. Table 2 shows the total system vehicle distance, vehicle time, and average speed for all demand modeled (truck and passenger car), a total of

TABLE 1 Total System Emissions for MOVES and Traffic Scenario Model Runs

\begin{tabular}{lrrr}
\hline Traffic Assignment & $\begin{array}{c}\text { TT Paths } \mathrm{CO}_{2} \\
\text { Emissions }(\mathrm{g})\end{array}$ & \multicolumn{1}{c}{$\begin{array}{l}\text { EMISS Paths } \mathrm{CO}_{2} \\
\text { Emissions (g) }\end{array}$} & Improvement (\%) \\
\hline MOVES_Car_and_Truck & $14,455,903,587$ & $14,367,090,051$ & 0.61 \\
Cube_Car_and_Truck & $425,522,530$ & $424,913,285$ & 0.14 \\
Cube_Just_Truck & $36,903,799$ & $36,886,838$ & 0.05 \\
\hline
\end{tabular}


TABLE 2 Total System Changes for the TT and Emissions Path Scenarios

\begin{tabular}{lllll}
\hline $\begin{array}{l}\text { Traffic } \\
\text { Assignment } \\
\text { Scenario }\end{array}$ & $\begin{array}{l}\text { Vehicle Distance } \\
(\mathrm{mi})\end{array}$ & $\begin{array}{l}\text { Vehicle } \\
\text { Time (h) }\end{array}$ & $\begin{array}{l}\text { Average } \\
\text { Speed } \\
(\mathrm{mph})\end{array}$ & $\begin{array}{l}\text { Total } \\
\text { Vehicles }\end{array}$ \\
\hline $\begin{array}{l}\text { TT paths } \\
\begin{array}{l}\text { Emissions } \\
\text { paths }\end{array}\end{array}$ & $14,008,913.55$ & $358,153.53$ & 39.11 & $1,753,942$ \\
\hline
\end{tabular}

$1,753,942$ vehicles. To reduce the total system's emissions, the average speed of all vehicles in the system was reduced by only $0.02 \mathrm{mph}$. The total vehicle miles traveled had to increase to $8,596.67$ and the total vehicle hours traveled needed to increase by $32.69 \mathrm{~h}$.

Additional investigation sought better understanding of the types of routes chosen for the two scenarios to learn what in the model prompted the reduced emissions. To understand the route changes, path analysis was used with observations of the demand on the modeled network with the path file for the two scenarios and identifying any major changes. Two types of truck travel were observed to see what kind of route changes occurred: regional external origin to external destination travel and local internal origin to internal origin.

Figures 4 and 5 show views of regional truck traffic. Figure 4 shows the results for the TT path scenario and Figure 5 shows the results for the emissions path scenario. A noticeable difference in routes can be seen by the use of the central bridge tunnel in the TT path scenario (Monitor-Merrimack Memorial Bridge-Tunnel) and use of the left bridge in the emissions path scenario (James River Bridge). Of the trucks that used the Monitor Merrimack crossing, $25 \%$ rerouted to the James River Bridge to reduce the total system emissions.

Figures 6 and 7 show views of local truck traffic, where Figure 6 shows the results for the TT path and Figure 7 shows the results for the emissions path scenario. A noticeable difference in routes can be seen by the use of the bridge to the right in the TT path scenario (Berkley Bridge) and the left tunnel by the emission path scenario (Midtown Tunnel). Of the trucks that used the Berkley Bridge crossing, $71 \%$ rerouted to the Midtown Tunnel to reduce the total system emissions.

Car paths changed very slightly in various parts of the road network, on freeways, arterials, and local roads. Large reroutes were not identified and only small ones occurred for the passenger cars throughout the system.

\section{CONCLUSION}

A simplified emissions calculation was used based on a binning method that used the information from the input tables in MOVES and an aggregate measure of VSP. This calculation was executed between the traffic assignment Frank-Wolfe iterations, providing an up-to-date value of emissions per segment. With an updated emissions value per segment, the path cost equation was amended to include emissions as an added cost to congested travel time. With the emissions plus travel time path cost, the model was run and tested with truck volume to see if it would converge. The results showed that emissions values were too large to allow good convergence, but

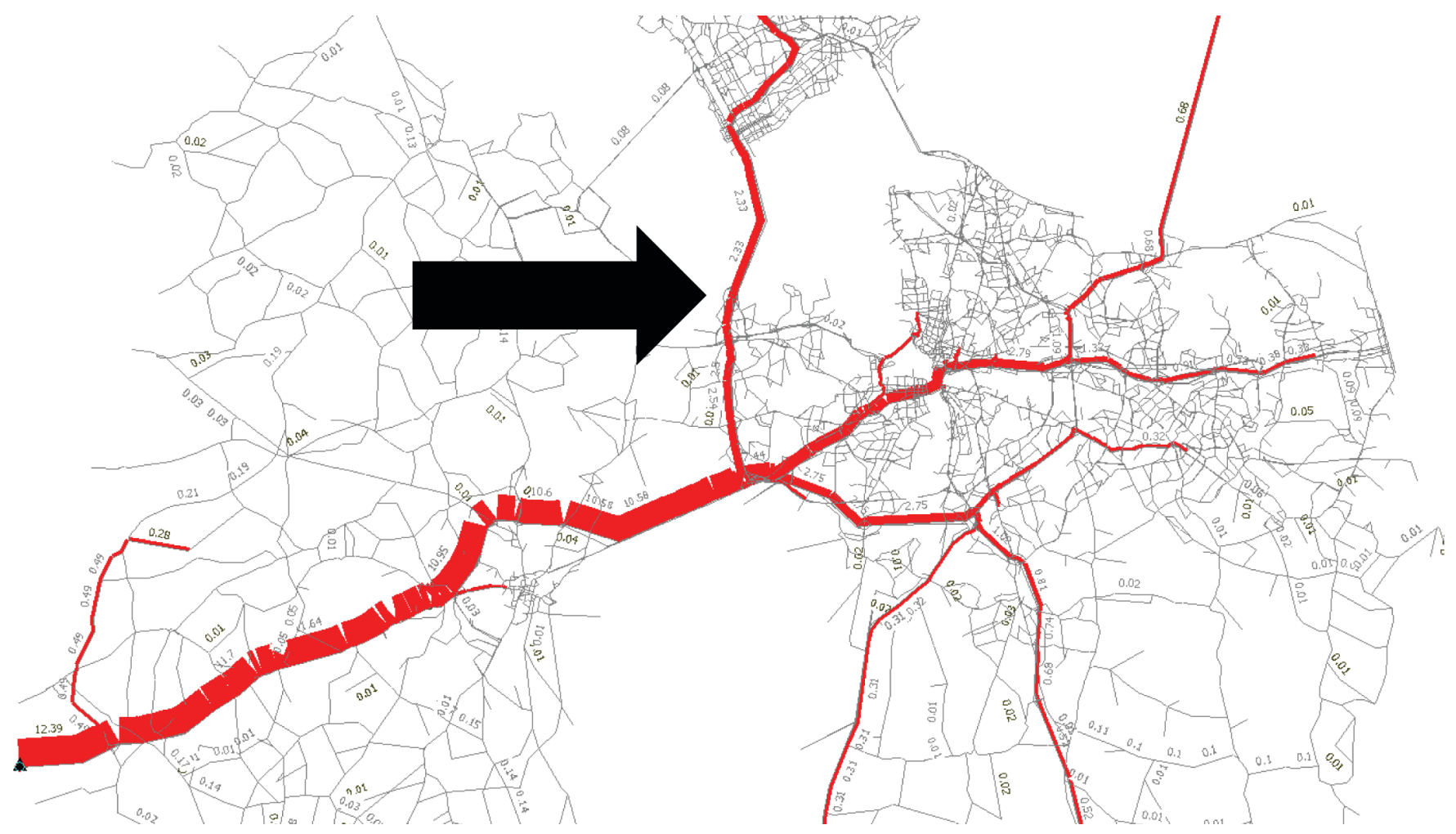

FIGURE 4 Regional truck travel for TT path scenario with noticeable use of central bridge tunnel (Monitor-Merrimack Memorial Bridge-Tunnel). 


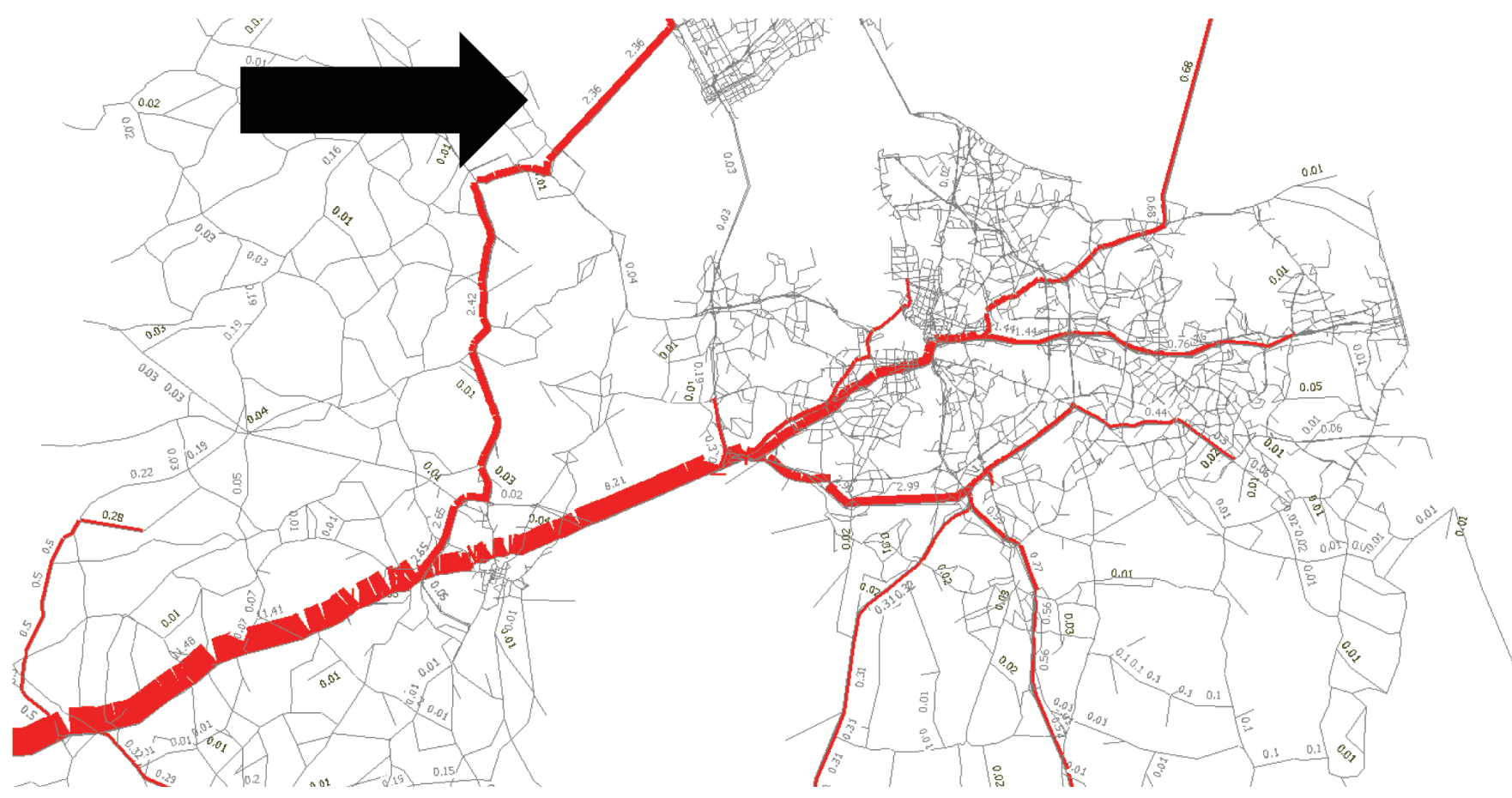

FIGURE 5 Regional truck travel for emissions path scenario with noticeable use of left bridge (James River Bridge).

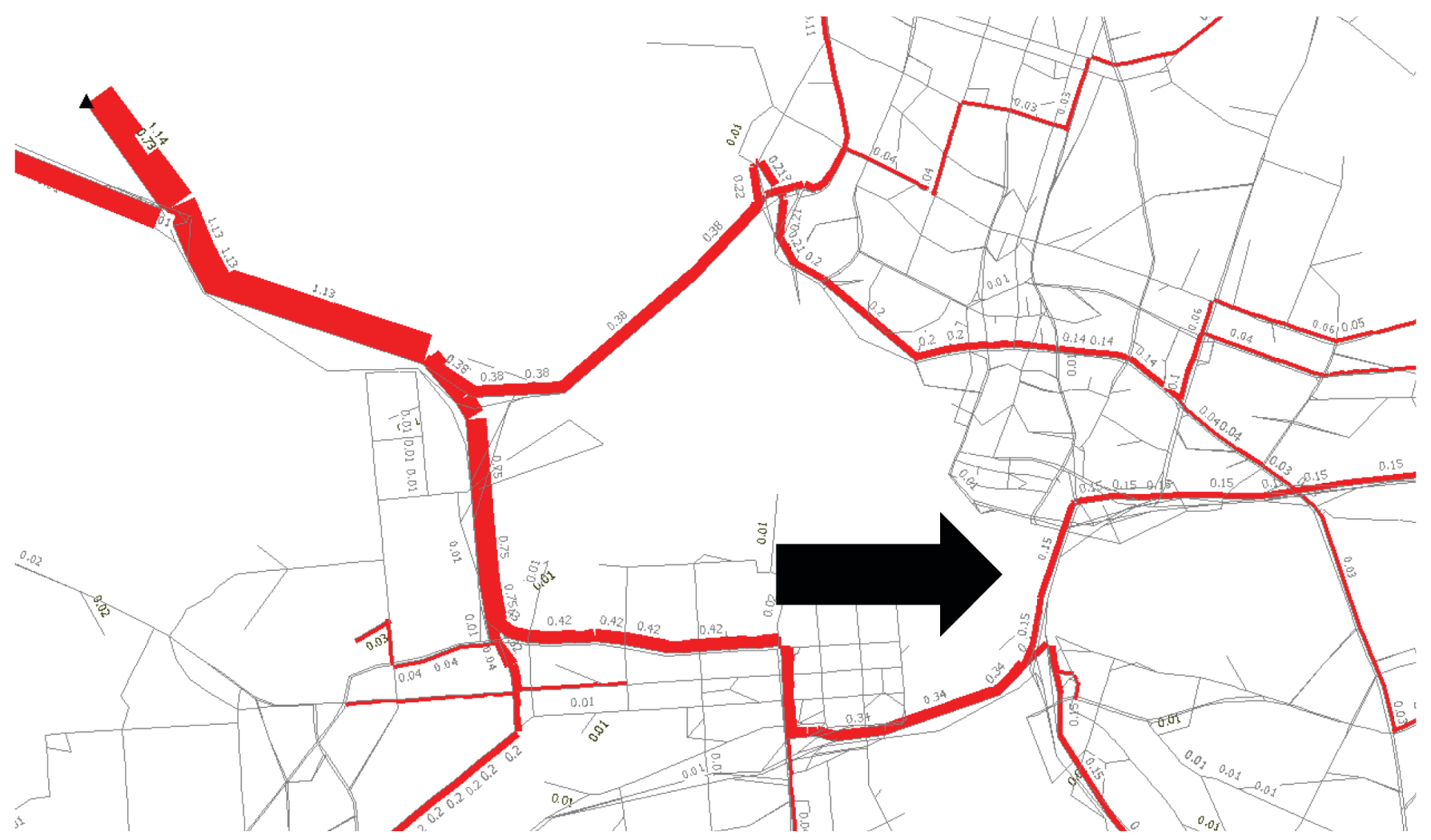

FIGURE 6 Local truck travel with noticeable use of bridge to right (Berkley Bridge). 


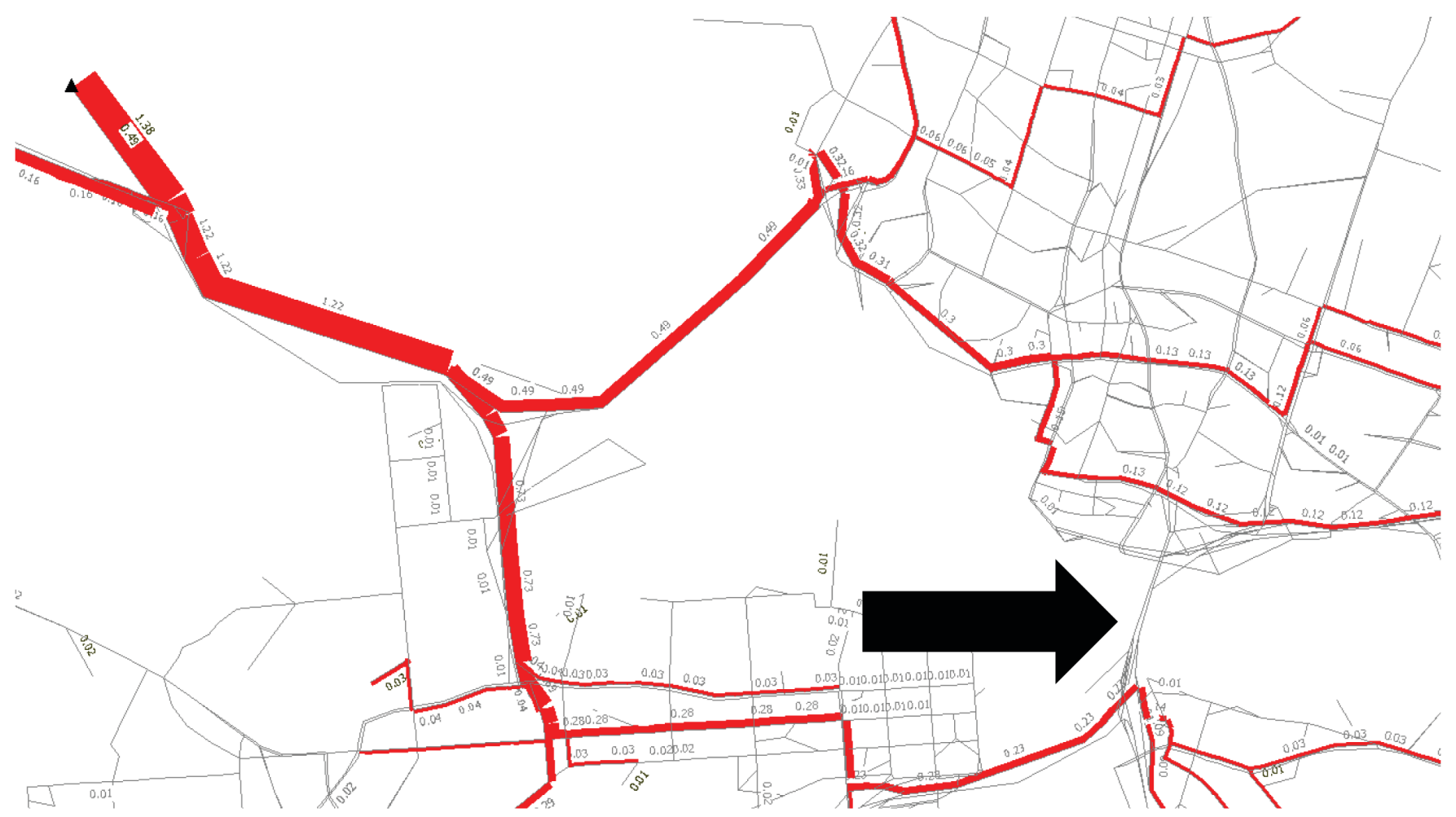

FIGURE 7 Local truck travel with noticeable lack of use of bridge to right (Berkley Bridge).

reducing the size of emissions by dividing the emissions values by a flat static value, the model produced results that converged.

The total truck emissions as calculated in the traffic assignment were recorded for a scenario where trucks used a path cost of emissions plus travel time and passenger cars used path costs of travel time only. Emissions were also recorded for a scenario where trucks and passenger cars used a path cost of travel time only. The results from both scenarios were run with the MOVES emissions model and compared. The methodology described was able to reduce total system truck emissions by a small percentage $(0.61 \%$, 88.8 tons), but showed that the methodology is capable of minimizing emissions. The results showed that reducing emissions came at essentially negligible cost to the total system TT and trucks changing routes did not impact passenger car travel. The area modeled showed that emissions produced by truck equilibrium where TT is reduced are very close to the equilibrium where emissions are reduced.

Studies such as this and others that utilize this methodology for truck traffic can help answer several questions. What regional emissions reductions can be achieved based on route choice? How will this operational decision affect TT? How do these decisions impact the routes and congestion of passenger cars? Although truck emissions reduction was not large in this scenario, the results are greatly dependent on the type of network used, such as the number of routes that can be used for rerouting, and the percentage of trucks compared with cars. The results could differ in other areas that utilize the same methodology. The possibility of other areas implementing this same methodology is great and results such as these can help planners better understand the potential improvements that can be achieved in the regional transportation system. Results from studies such as this can be beneficial to decision makers who intend to improve emissions for a region through changes in routing.

\section{FUTURE WORK}

To extend this research, future work will require improving the VSP calculation. Additional influences, including road grade, rolling resistance, and wind resistance values, should be considered. Implementing these values, especially grade changes, may have a great effect on what routes are chosen to reduce emissions. The acceleration value is an aggregate value and would be more accurate when measured from a microscopic model. Regional microscopic models are becoming more viable and the work done in this study compliments the future of transportation modeling. Large microscopic models will need to utilize dynamic traffic assignment algorithms to determine the distribution of flows and the same methodology can be applied to those models but with a better measure of VSP.

\section{ACKNOWLEDGMENTS}

Funding for this study was provided by TranLIVE through the U.S. Department of Transportation University Transportation Centers Tier 1 award.

\section{REFERENCES}

1. Greene, D.L., and S.E. Plotkin. Reducing Greenhouse Gas Emissions from U.S. Transportation. Pew Center on Global Climate Change, 2011. 
2. Dablanc, L., G. Giuliano, K. Holliday, and T. O’Brien. Best Practices in Urban Freight Management: Lessons from an International Survey. In Transportation Research Record: Journal of the Transportation Research Board, No. 2379, Transportation Research Board of the National Academies, Washington, D.C., 2013, pp. 29-38.

3. Bühler, G., and P. Jochem. $\mathrm{CO}_{2}$ Emission Reduction in Freight Transports: How to Stimulate Environmental Friendly Behaviour. Presented at 88th Annual Meeting of the Transportation Research Board, Washington, D.C., 2009.

4. Yan, X., and R.J. Crookes. Reduction Potentials of Energy Demand and GHG Emissions in China's Road Transport Sector. Energy Policy, Vol. 37, 2009, pp. 658-688.

5. Li, Y., L. Bao, and J. Bao. Evaluating the Emission Mitigation Potential of Shanghai Transportation Policies Using LEAP Model. Presented at 93rd Annual Meeting of the Transportation Research Board, Washington, D.C., 2014.

6. Pietz, A. J., and B. J. Gregor. Oregon Statewide Transportation Strategy: 2050 Vision for Greenhouse Gas Emission Reduction. In Transportation Research Record: Journal of the Transportation Research Board, No. 2454, Transportation Research Board of the National Academies, Washington, D.C., 2014, pp. 45-52.

7. Bhagat, A., J.-D. Saphores, R. Jayakrishnan, and J. Jung. An Analysis of Air Pollution Impacts from PierPASS in the Alameda Corridor,
California. Presented at 93rd Annual Meeting of the Transportation Research Board, Washington, D.C., 2014.

8. McKinnon, A.C. Benchmarking Road Freight Transport: Review of a Government-Sponsored Programme. Benchmarking: An International Journal, Vol. 16, No. 5, 2009, pp. 640-656.

9. Rowell, M., A. Gagliano, Z. Wang, A. Goodchild, J. Sage, and E. Jessup. Improving Statewide Freight Routing Capabilities for Sub-National Commodity Flows. Final Research Report WA-RD-792.1, 2012. http:// www.wsdot.wa.gov/research/reports/fullreports/792.1.pdf. Accessed July $16,2014$.

10. Ahn, K., and H. Rakha. The Effects of Route Choice Decisions on Vehicle Energy Consumption and Emissions. Transportation Research Part D, Vol. 13, 2008, pp. 151-167.

11. Cube. Citilabs Inc., Lafayette, Calif., 2012.

12. Urban Mass Transportation Administration. UMTA Transportation Planning System Reference Manual. National Technical Information Service, Alexandria, Va., 1977.

The views expressed are the authors'

The Standing Committee on Transportation and Air Quality peer-reviewed this paper. 